\title{
A Data-driven Vessel Motion Model for Offshore Access Forecasting
}

\author{
Ciaran Gilbert \\ Electronic \& Electrical Engineering \\ University of Strathclyde \\ Glasgow, UK \\ ciaran.gilbert@strath.ac.uk
}

\author{
Jethro Browell \\ Electronic \& Electrical Engineering \\ University of Strathclyde \\ Glasgow, UK \\ jethro.browell@strath.ac.uk
}

\author{
David McMillan \\ Electronic \& Electrical Engineering \\ University of Strathclyde \\ Glasgow, UK \\ d.mcmillan@strath.ac.uk
}

\begin{abstract}
Access forecasting for offshore wind farm operations is concerned with the prediction of conditions during transfer of personnel between offshore structures and vessels. Currently dispatch/scheduling decisions are typically made on the basis of single-valued forecasts of significant wave height from a numerical weather prediction model. The aim of this study is to move beyond the significant wave height metric using a datadriven methodology to estimate vessel motion during transfer. This is because turbine access is constrained by the behaviour of crew transfer vessels and the transition piece in the local wave climate. Using generalised additive models for location, scale, and shape, we map the relationship between measured vessel heave motion and measured wave conditions in terms of significant wave height, peak wave period, and peak wave direction. This is explored via a case study where measurements are collected via vessel telemetry and an on-site wave buoy during the construction phase of an east coast offshore wind farm in the UK. Different model formulations are explored and the best performing trained model, in terms of the Akaike Information Criterion, is defined. Operationally, this model is driven by temporal scenario forecasts of the input wave buoy measurements to estimate the vessel motion during transfer up to 5 days ahead.
\end{abstract}

Index Terms-offshore wind, offshore operations, offshore access, additive models, data-driven, forecasting

\section{INTRODUCTION}

Offshore wind power is now a major source of supply in European electricity markets. With the expected growth of the market in the next few years and the possibility of exploiting deeper waters, access to the turbines is crucially important in the construction and operation \& maintenance (O\&M) phases of the wind farm life cycle [1]. Access will also be important as assets in the water age and components need to be replaced. Improving access is a route to reducing the cost of wind energy by reducing turbine downtime, and improved operations attractive due to the prospect of savings being made at any time and across the remaining life of the wind farm, and that these savings can be realised independently of turbine manufacturers.

Access forecasting is concerned with the prediction of the conditions for transfer of personnel between offshore structures and vessels at the wind farm. In practice, this

This work is supported by the EPSRC Supergen Wind Hub project ORACLES, EP/L014106/1.

978-1-7281-1450-7/19/\$31.00 @2019 IEEE currently translates as single-valued deterministic forecasts of significant wave height obtained via Numerical Weather Prediction (NWP) models for horizons of over approximately 6 hours [2], [3]. This information is assimilated along with information flows from assets, other important weather variables (lightning risk, visibility, etc), and live point measurements by the marine coordination team who then use this information, the current schedule, and site expertise to execute operation and maintenance each day. The skipper, crew, and technicians on each Crew Transfer Vessel (CTV) also form a pivotal part of the decision making chain as they have the final say on any attempted transfer.

Typically, the scheduling and dispatch process is coupled with a service contract where transfers are expected to be attempted below a significant wave height threshold, typically around $1.5 \mathrm{~m}$ for CTVs [1]. However, access is constrained by the interaction of the vessel and the turbine access point. Therefore, this study aims to go beyond the typical deterministic significant wave height forecast by using a datadriven vessel motion model. Operationally, this translates input forecasts of wave height, period, and direction into actionable information for the end-user based on the expected vessel motion during transfer. Additionally, probabilistic forecasting is explored to quantify the uncertainty in the weather forecasts. The economic benefit of using probabilistic forecasts for offshore operations is explored in [4].

Vessel scheduling and dispatch is a form of time-dependent decision making; consider that good access conditions at a single point in time is not sufficient as technicians must complete work and be collected by the CTV at a later time. Therefore, to usefully predict the quality of transfer over the duration of the forecast horizon the uncertainty must be accounted for at each step and be linked between horizons. This stems from meteorological forecast error persistence, i.e. if the significant wave height is over-forecast at a specific timestep, then it is likely to be over-forecast at the adjacent timestep. Statistical scenario forecasts (or trajectories) are used in these applications [5]. Scenario forecasts are closely related to meteorological physical ensemble forecasts which is a staple of the operational NWP community, and could equally be used to support time-dependent decision making [6], although the number of scenarios is limited and site-specific re-calibration 
would be necessary [7]. The goal of this paper is to evaluate the process of converting these met-ocean scenario forecasts into vessel-specific forecast scenarios of motion during transfer.

This application therefore requires a model of the vessel motion during transfer events; such models can be classified into physical models of the vessel motion or a data-driven approach, the latter being the focus of this paper. The physical modelling approach is explored in [8]-[11] whereby variations of hydrodynamic models of the vessel/access point are constructed. This has the advantage of fully characterising the vessel response in the wave climate of interest across the 6 degrees of motion. However, requires an input forecast seastate spectrum to excite the model in an operational forecasting environment. An alternative is a fully data-driven model, whereby using vessel telemetry, tagged transfer attempts, and concurrent sea-state point measurements a model of the heave motion of the vessel during transfer can be constructed. Operationally, forecasts of summary statistics of sea-state variables can then be used to drive the vessel motion model.

In this study, to construct the data-driven model of vessel motion during transfer, generalised additive models are used [12] which enable the mapping of vessel heave measurements to additive functions of wave buoy measurements. In this instance understanding the uncertainty around transfer conditions is essential, as well as modelling under heteroscedasticity to fully capture the relationship; consider that under transferring at the edge of the vessel capabilities leads to more uncertainty in the corresponding vessel motion. A class of regression models that are capable of dealing with this, as well as the non-negative nature of heave motion, are Generalised Additive Models for Location, Scale, and Shape [13].

This paper is organised as follows: Section II briefly describes the method for generating met-ocean scenario forecasts, Section III introduces the regression tools for the datadriven vessel model, followed by Section IV where the data used is introduced, results are presented for the case study, and future work is outlined. Conclusions are drawn in Section V.

\section{Generating Met-Ocean Forecast Scenarios}

This section briefly outlines the methodology required to obtain operational inputs for the vessel motion model in the access forecasting tool. For more information on the entire forecasting methodology the reader is referred to related work by the authors [14]. The block flow diagram in Figure 1 illustrates the modelling stages of the access forecasting tool, as well as the streams of data used in both the operational and training stage of the modelling.

Site-specific post-processing of the weather forecast data is used to increase the accuracy of the forecasts and quantify the uncertainty for each variable of interest (significant wave height, peak wave period, and peak wave direction). This stage improves accuracy by removing systematic biases present in the weather forecasts for the specific location of interest, which are a feature of NWP output in general when comparing

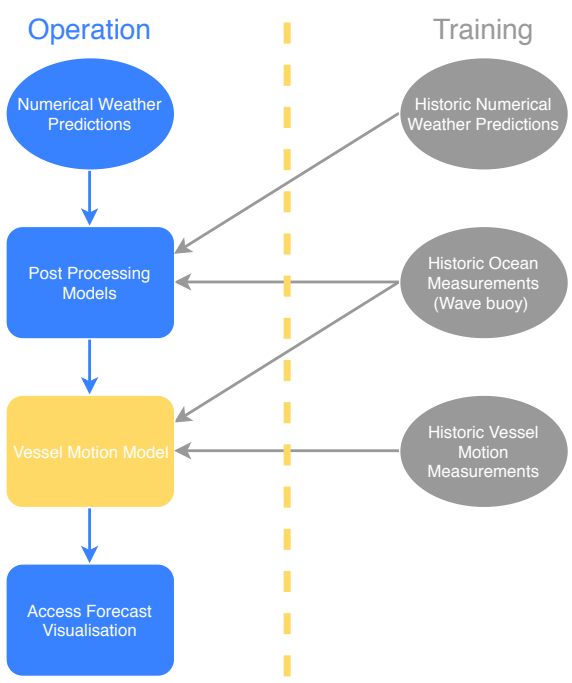

Fig. 1: Flowchart illustration of the access forecasting modelling chain in operation and training phases. The vessel motion model aspect (coloured gold) is the focus of this paper

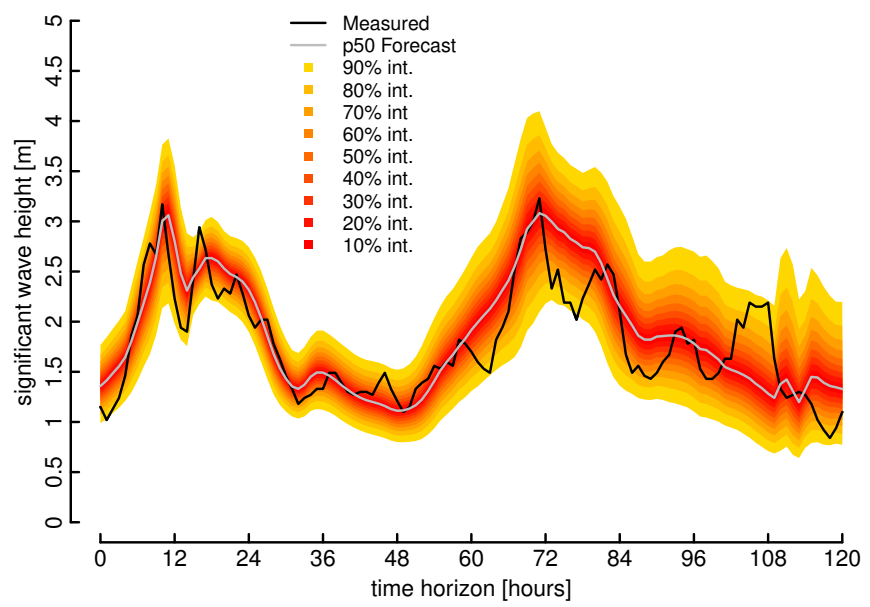

Fig. 2: Example post-processed density forecast of significant wave height up to 5 days ahead

to specific measurement locations. Both quantile regression and parametric distribution regression are explored to map the relationship between the respective measurements at the wave buoy and concurrent historical NWPs. An example of a probabilistic density forecast of significant wave height for up to 5 days ahead is shown in Figure 2.

To generate the statistical scenario forecasts, the joint distribution of each time horizon must be estimated using the marginal distributions (i.e. Figure 2) and a copula function [5]. Samples with the correct temporal dependency structure can then be drawn from the high-dimensional distribution and used operationally as temporal scenarios for the vessel motion model, which is the focus of this paper. The corresponding scenario forecast of significant wave height is shown in Figure 3. 


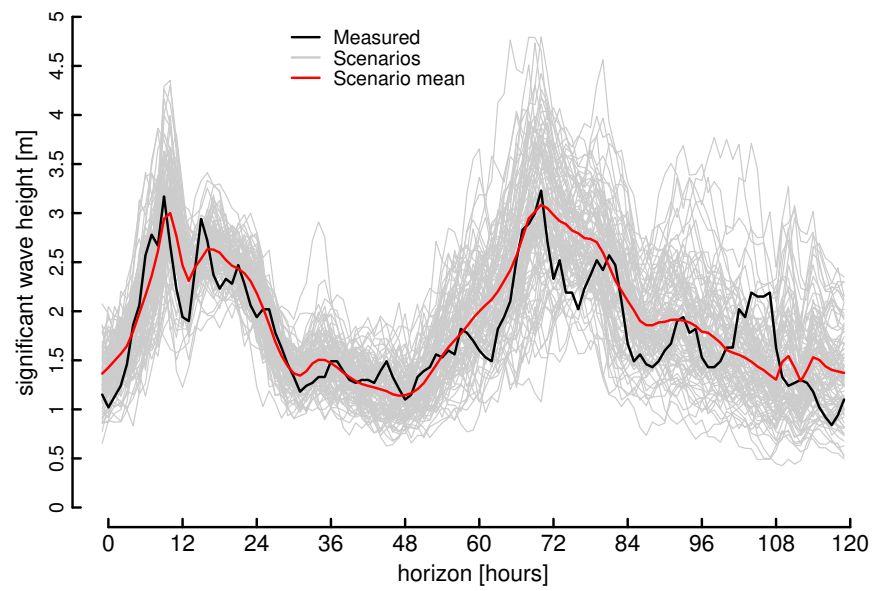

Fig. 3: Example post-processed scenario forecast of significant wave height up to 5 days ahead

Wave direction is incorporated in the modelling in a straightforward manner by first clustering the measured data to separate distinct sea states. The sea-states regimes are dominated either locally generated wind waves or swell from the North Sea. Weather forecasts are then used to predict the probability of cluster membership via logistic regression. This completes the operational input variable space for the access forecasting tool which consist of temporal scenario forecasts of significant wave height and peak wave period, as well as categorical forecasts of sea-state regimes. For training of the vessel motion model corresponding measurements of the above are used, i.e. significant wave height $H_{s}$, peak wave period $T_{p}$, and clustered sea-state regimes reg. which are dominated by peak wave direction $\omega_{p}$.

\section{Generalised Additive Models for Location, SCALE, AND SHAPE}

This section details the regression model used to estimate vessel motion during transfer. Generalised Additive Models for Location, Scale, and Shape (gamlss) [13] models are termed semi-parametric regression models. They are semi-parametric in that an assumed conditional distribution is defined in the model, however the parameters which govern the distribution may include non-parametric smoothing functions in the regression. This framework is an expansion of the more familiar generalised additive models [12] in that any parameter of the distribution can be a function of the explanatory variables, not just the conditional mean.

Consider a target variable $y$, in this case vessel heave motion, the conditional density $f_{d}(y \mid \boldsymbol{\theta})$ depends on up to four parameters; these are the location $\left(\theta_{1}\right)$, scale $\left(\theta_{2}\right)$, and shape parameters $\left(\theta_{3}, \theta_{4}\right)$, where the latter are normally referred to as skewness and kurtosis parameters of the distribution. An additive regression model $\eta_{\theta_{i}}$ is created for each distribution parameter $\theta_{i}$ for $i=1, \ldots, 4$. Let $\boldsymbol{x}_{i}$ be the pool of $N_{i}$ explanatory variables in the submodel for $\theta_{i}$, and $g_{i}($.$) is the$ defined link function, then the model formulation of gamlss is

$$
g_{i}\left(\theta_{i}\right)=\eta_{\theta_{i}}=\beta_{0 \theta_{i}}+\sum_{n=1}^{N_{i}} f_{n \theta_{i}}\left(x_{i n}\right), \quad i=1, \ldots, 4
$$

where the function $f_{n \theta_{i}}$ is the effect of explanatory variable $n$ on the distribution parameter $\theta_{i}$, which can be linear or nonlinear effects; $\beta_{0 \theta_{i}}$ are the intercepts of each submodel. These models are fitted iteratively using a combination of maximum likelihood, transformation of distribution parameters $\boldsymbol{\theta}$ using the inverse link function, and successive backfitting of the predictor functions in each submodel $\eta_{\theta_{i}}$. For more information, the reader is referred to [13].

\section{CASe Study}

This section introduces the data available for the case study, results, as well as discussion points throughout the analysis, and future work. The case study is carried out at an east coast offshore wind farm in the UK; the vessel telemetry data is collected during the construction phase of the wind farm from two purpose built offshore wind service vessels with the same specification: length $19.2 \mathrm{~m}$, width $8.2 \mathrm{~m}$, maximum Draft $2 \mathrm{~m}$, passengers 12 , aluminium catamaran. Ocean measurements are collected from a Centre of Environment Fisheries and Aquaculture Science (CEFAS) wave-buoy within the site boundary. The method is implemented in $\mathrm{R}$ using the package gamlss [13], [15].

Approximately 700 push-on instances are available alongside concurrent wave buoy measurements. To label transfer events the measured push-on force is available from the vessel telemetry, as well as time-stamped swipes from personnel ID cards following any transfer event. For matching the time resolution of the vessel motion and buoy measurements, the closest buoy measurement in time is used and all the vessel measurements are averaged per push-on event. The focus of this analysis is the vessel heave peak to peak measurement, which is a measure of the vertical displacement of the vessel, localised to the area of measurement, during transfer events.

To test different model configurations a goodness-of-fit metric is used, called the Akaike Information Criterion (AIC)

$$
\mathrm{AIC}=2 k-\ln (\hat{L})
$$

which rewards the model with the highest likelihood function $\hat{L}$, penalised by the number of input parameters $k$ used to estimate the model; overfitting is then less likely for the model with the minimum AIC.

To develop the model several configurations are tested, beginning with basic linear regression models and leading to truncated regression models with smooth non-parametric base learners. As discussed, truncated regression is deployed to respect the non-negativity of the vessel heave peak to peak measurements. Results of the case study are detailed in Table I, which shows that eventually using a truncated student$t$ distribution as the parametric response family allows for better AIC results compared to the normal distribution family; 
TABLE I: Formulations for the vessel motion model during transfer dependent on observed sea-state. The symbols $p s$ indicates a penalised beta spline, $\dagger$ is a varying coefficient model, poly is a fractional polynomial model, $H_{s}$ is significant wave height, $T_{p}$ is peak wave period, $\omega_{p}$ is peak wave direction, and reg. is the regime membership.

\begin{tabular}{|c|c|c|c|c|c|}
\hline ID & Distribution & Formula \& Expla & $\begin{array}{l}\text { tory Variables } \\
\text { Scale }\end{array}$ & Shape & AIC \\
\hline $\mathrm{N}-1$ & Normal & $\sim H_{s}$ & $\sim 1$ & $\mathrm{n} / \mathrm{a}$ & -1653 \\
\hline $\mathrm{N}-2$ & Normal & $\sim H_{s}+T_{p}$ & $\sim 1$ & $\mathrm{n} / \mathrm{a}$ & -1687 \\
\hline $\mathrm{N}-3$ & Normal & $\sim H_{s}+T_{p} * H_{s}$ & $\sim 1$ & $\mathrm{n} / \mathrm{a}$ & -1695 \\
\hline $\mathrm{N}-4$ & Normal & $\sim H_{s}$ & $\sim H_{s}$ & $\mathrm{n} / \mathrm{a}$ & -1746 \\
\hline N-5 & Normal & $\sim H_{s}+T_{p}$ & $\sim H_{s}$ & $\mathrm{n} / \mathrm{a}$ & -1779 \\
\hline N-6 & Normal & $\sim H_{s}+T_{p} * H_{s}$ & $\sim H_{s}$ & $\mathrm{n} / \mathrm{a}$ & -1787 \\
\hline $\mathrm{N}-7$ & Normal & $\sim H_{s}+\operatorname{poly}\left(T_{p}+T_{p}^{2}\right)$ & $\sim H_{s}$ & $\mathrm{n} / \mathrm{a}$ & -1806 \\
\hline $\mathrm{N}-8$ & Normal & $\sim H_{s}+\operatorname{poly}\left(T_{p}+T_{p}^{2}\right)$ & $\sim H_{s}+\mathbf{1}\left(\omega_{p}<120\right)$ & $\mathrm{n} / \mathrm{a}$ & -1811 \\
\hline $\operatorname{tr}-\mathrm{N}-1$ & Tr. Normal $[0, \infty)$ & $\sim H_{s}+\operatorname{poly}\left(T_{p}+T_{p}^{2}\right)$ & $\sim H_{s}+\mathbf{1}\left(\omega_{p}<120\right)$ & $\mathrm{n} / \mathrm{a}$ & -1823 \\
\hline $\mathrm{T}-1$ & $t$-family & $\sim H_{s}+\operatorname{poly}\left(T_{p}+T_{p}^{2}\right)$ & $\sim H_{s}+\mathbf{1}\left(\omega_{p}<120\right)$ & $\sim 1$ & -1811 \\
\hline $\operatorname{tr}-\mathrm{T}-1$ & Tr. $t$-family $[0, \infty)$ & $\sim H_{s}+\operatorname{poly}\left(T_{p}+T_{p}^{2}\right)$ & $\sim H_{s}+\mathbf{1}\left(\omega_{p}<120\right)$ & $\sim 1$ & -1823 \\
\hline$t r-\mathrm{T}-2$ & Tr. $t$-family $[0, \infty)$ & $\sim H_{s}+\operatorname{poly}\left(T_{p}+T_{p}^{2}\right)$ & $\sim H_{s}+\mathbf{1}\left(\omega_{p}<120\right)$ & $\sim H_{s}$ & -1827 \\
\hline $\operatorname{tr}-\mathrm{T}-3$ & Tr. $t$-family $[0, \infty)$ & $\sim H_{s}+\operatorname{ps}\left(T_{p}\right)$ & $\sim H_{s}+\mathbf{1}\left(\omega_{p}<120\right)$ & $\sim H_{s}$ & -1829 \\
\hline $\operatorname{tr}-\mathrm{T}-4$ & Tr. $t$-family $[0, \infty)$ & $\sim H_{s}+\operatorname{ps}\left(T_{p}\right)$ & $\sim H_{s}+$ reg. & $\sim H_{s}$ & -1826 \\
\hline $\operatorname{tr}-\mathrm{T}-5$ & Tr. $t$-family $[0, \infty)$ & $\sim \operatorname{ps}^{\dagger}\left(H_{s}\right.$, reg. $)+\operatorname{ps}\left(T_{p}\right)$ & $\sim H_{s}+$ reg. & $\sim 1$ & -1830 \\
\hline $\operatorname{tr}-\mathrm{T}-6$ & Tr. $t$-family $[0, \infty)$ & $\sim \operatorname{ps}^{\dagger}\left(H_{s}\right.$, reg. $)+\operatorname{ps}\left(T_{p}\right)+T_{p} * H_{s}$ & $\sim H_{s}+$ reg. & $\sim 1$ & -1838 \\
\hline $\operatorname{tr}-\mathrm{T}-7$ & Tr. $t$-family $[0, \infty)$ & $\sim \operatorname{ps}^{\dagger}\left(H_{s}\right.$, reg. $)+\operatorname{ps}\left(T_{p}\right)+T_{p} * H_{s}$ & $\sim \operatorname{ps}^{\dagger}\left(H_{s}\right.$, reg. $)$ & $\sim 1$ & -1846 \\
\hline
\end{tabular}

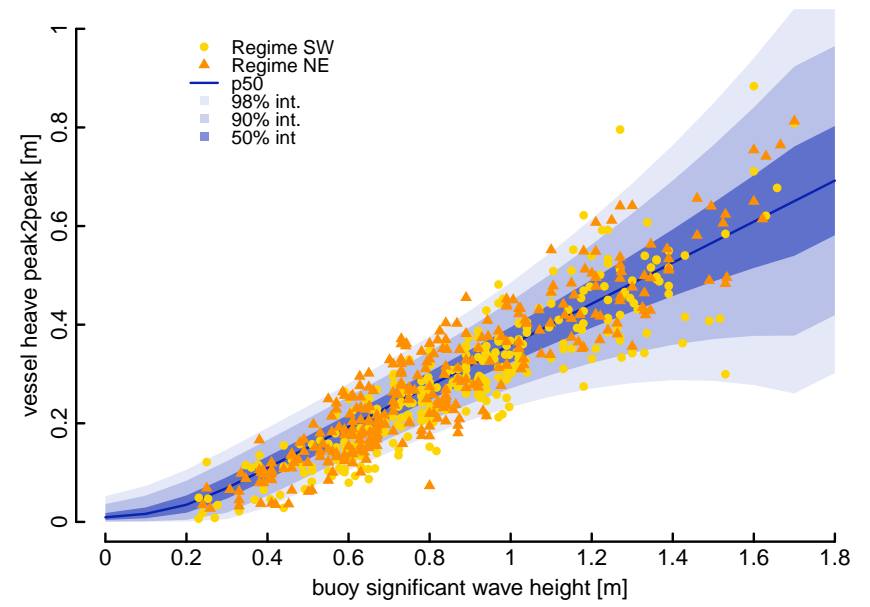

(a) Model fit in South-Westerly (SW) regime

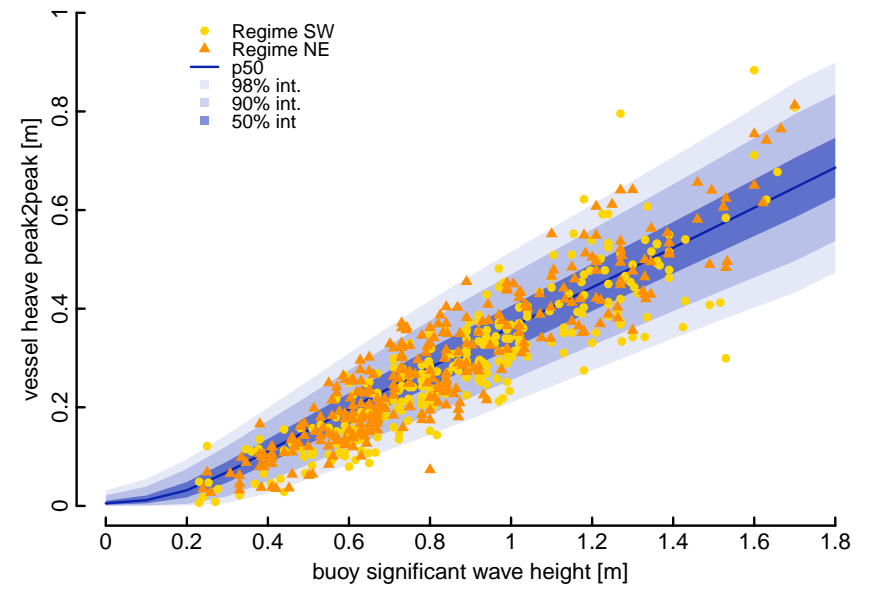

(b) Model fit in North-Easterly (NE) regime

Fig. 4: Plot of vessel motion during push-on and concurrent ocean measurements. The distributional model fit is for the $t r-\mathrm{T}-7$ model with constant peak wave period

this is due to the ability to specify the shape parameter of the distribution.

In terms of the base-learner functions, improvement is also indicated when using polynomial functions compared to linear effects, as shown in Table I. Eventually penalised beta splines are employed for modelling the effect of significant wave height and peak wave period on both the location and scale parameters. Additionally, modelling the interaction of both variables is clearly valuable for the location of the distribution. Incorporating peak wave direction also improves the AIC results via influencing the scale parameter of the distribution, either through an indicator variable or through the clustered regime membership.

The marginal effect plot of significant wave height is shown in Figure 4 for the minimum AIC model ( $t r-\mathrm{T}-7)$ with peak period held constant. The model formulation means that under the different regimes the coefficients of the penalised spline learner for $H_{s}$ change for both the locations and scale parameters of the distribution. The effect of the varying coefficient spline is clear in the changing shape of the uncertainty between Figures $4 \mathrm{a}$ and $4 \mathrm{~b}$. This allows for the regime membership to influence the model fit more flexibly than simply varying the intercept terms. Lastly, the motivation behind using a model framework with conditional heteroscedasticity is clear, as the 


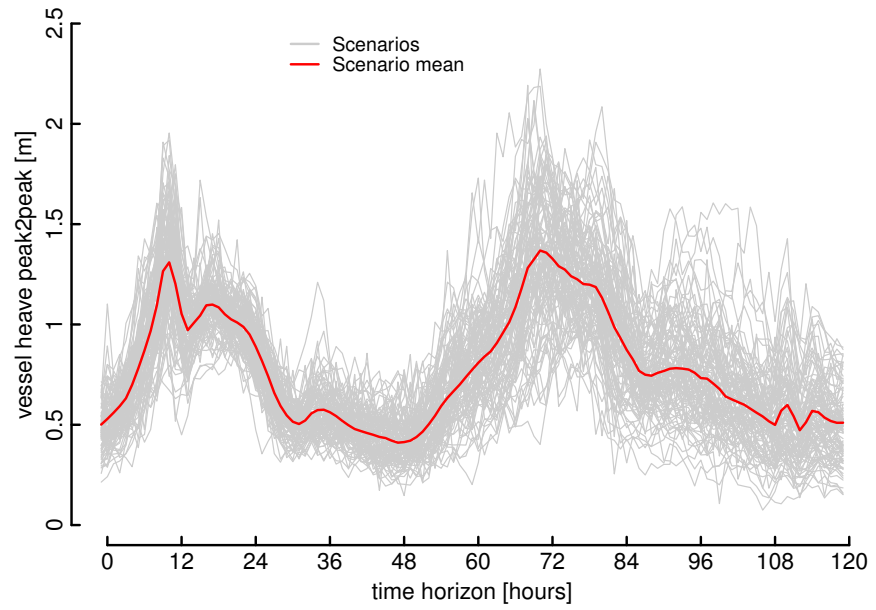

Fig. 5: Example scenario forecast of vessel motion during transfer up to 5 days ahead

uncertainty grows with significant wave height.

The final part of analysis includes an exploration of the operational characteristics of the vessel motion during transfer model. This means driving the model with the met-ocean forecast scenarios of wave height and period, as well as the forecast regime membership. A resulting example forecast is shown in Figure 5 where the mean heave peak to peak displacement is calculated for each input scenario. An important aspect to consider here is that the forecast significant wave height, which is obviously the major driver in the output of the vessel motion model when compared to Figure 3, is that often the forecast significant wave height is beyond the maximum measured wave height during transfer. Therefore, for the shape parameter of the model a threshold significant wave height feature is in practice used to stabilise predictions outside the region that is observed during training of the model. Forecasts must then be transformed from Figure 5 to reflect that there is zero chance of a successful transfer in this region, which is part of the motivation behind a related study on access forecast visualisation [16].

Future work should consider the utilisation of the uncertainty information in the vessel motion during transfer model, as only the mean displacement is considered. This could be done via sampling of the distribution or via a mixture model to generate a forecast density of heave displacement. However, computational expenditure may become an issue, especially using sampling, as the number of forecast scenarios can be prohibitive. Additionally, a turbine specific access model could be derived given a large enough dataset. The above model considers the three most important driving environmental factors, other site-specific driving environmental factors could be incorporated into the model such as tidal effects and visibility. Lastly, this model considers the motion of the vessel at the point of measurement and a useful extension would be to derive the motion of the vessel fender at the point of contact to the turbine transition piece.

\section{Conclusions}

This paper describes a data-driven methodology for moving access prediction for offshore operations beyond the typical significant wave height metric. To this end, the relationship between vessel motion during transfer and concurrent ocean measurements is mapped using a statistical learning technique. This unique dataset stems from vessel telemetry which measures heave peak-to-peak displacement and an on-site wave buoy measuring peak wave period, peak wave direction, and significant wave height. Using generalised additive models for location, scale, and shape different model formulations are explored via a case study, where measurements are collected during the construction phase of an east coast offshore wind farm in the UK. It is demonstrated that a truncated regression using penalised spline base-learners, interaction terms, and varying coefficient terms are capable of learning the desired relationship. Operationally, this model is driven with temporal scenario forecasts of the sea state to estimate the vessel motion during transfer up to 5 days ahead. This new forecasting capability pertains to offshore planners, schedulers, marine coordinators, and vessel skippers who will be able to make more informed safety-critical decisions, with the potential for reductions in the cost of offshore wind energy.

\section{ACKNOWLEDGMENT}

The authors would like to thank Amine Hadjer, John Best, and Pete Leach at James Fisher Marine Services, Lars Johanning and Giovanni Rinaldi at Exeter University, Sally Shenton at Generating Better, and Christopher Briggs at SiemensGamesa for their excellent discussion points and feedback throughout this work. Data Statement: Due to confidentiality agreements with research collaborators, access to vessel motion data is restricted. Wave buoy measurements from the Centre of Environment Fisheries and Aquaculture Science (CEFAS) are available at http://wavenet.cefas.co.uk/.

\section{REFERENCES}

[1] B. Maples, G. Saur, M. Hand, R. Van De Pietermen, and T. Obdam, "Installation, Operation, and Maintenance Strategies to Reduce the Cost of Offshore Wind Energy," National Renewable Energy Laboratory (NREL), Tech. Rep., 2013.

[2] V. M. Catterson, D. McMillan, I. Dinwoodie, M. Revie, J. Dowell, J. Quigley, and K. Wilson, "An economic impact metric for evaluating wave height forecasters for offshore wind maintenance access," Wind Energy, vol. 19, no. 2, pp. 199-212, 22016.

[3] G. Reikard, P. Pinson, and J. R. Bidlot, "Forecasting ocean wave energy: The ECMWF wave model and time series methods," Ocean Engineering, vol. 38, no. 10, pp. 1089-1099, 2011.

[4] J. W. Taylor and J. Jeon, "Probabilistic forecasting of wave height for offshore wind turbine maintenance," European Journal of Operational Research, vol. 267, no. 3, pp. 877-890, 62018.

[5] P. Pinson, H. Madsen, G. Papaefthymiou, and B. Klöckl, "From Probabilistic Forecasts to Statistical Scenarios of Short-term Wind Power Production," Wind Energy, vol. 12, no. 1, pp. 51-62, 2009.

[6] R. Bessa, C. Möhrlen, V. Fundel, M. Siefert, J. Browell, S. Haglund El Gaidi, B.-M. Hodge, U. Cali, and G. Kariniotakis, "Towards Improved Understanding of the Applicability of Uncertainty Forecasts in the Electric Power Industry," Energies, vol. 10, no. 9, p. 1402, 2017.

[7] P. Pinson and R. Girard, "Evaluating the quality of scenarios of shortterm wind power generation," Applied Energy, vol. 96, pp. 12-20, 2012. 
[8] M. Wu, "Numerical analysis of docking operation between service vessels and offshore wind turbines," Ocean Engineering, vol. 91, pp. 379-388, 112014.

[9] R. Guanche, M. Martini, A. Jurado, and I. J. Losada, "Walk-to-work accessibility assessment for floating offshore wind turbines," Ocean Engineering, vol. 116, pp. 216-225, 42016.

[10] M. König, D. F. González, M. Abdel-Maksoud, and A. Düster, "Numerical investigation of the landing manoeuvre of a crew transfer vessel to an offshore wind turbine," Ships and Offshore Structures, vol. 12, 3 2017.

[11] D. Ferreira González, M. Lemmerhirt, M. Abdel-Maksoud, M. König, and A. Düster, "Numerical and Experimental Investigation Regarding the Landing Manoeuvre of a Catamaran Vessel at an Offshore Wind Turbine in Waves," in International Conference on Offshore Mechanics and Arctic Engineering, Volume 7: Ocean Engineering. Newfoundland, Canada: ASME, 2015.

[12] T. Hastie, R. Tibshirani, and J. H. Friedman, The Elements of Statistical Learning: Data Mining, Inference, and Prediction, 2nd ed. Springer, 2009.

[13] R. A. Rigby, D. M. Stasinopoulos, and P. W. Lane, "Generalized additive models for location, scale and shape," Journal of the Royal Statistical Society. Series C: Applied Statistics, vol. 54, no. 3, pp. 507-554, 62005.

[14] C. Gilbert, J. Browell, and D. McMillan, "Probabilistic Access Forecasting for Offshore Operations," [in preparation], 2019.

[15] R Core Team, "R: A Language and Environment for Statistical Computing," Vienna, Austria, 2016. [Online]. Available: https://www.rproject.org/

[16] C. Gilbert, J. Browell, and D. McMillan, "Visualisation of Probabilistic Access Forecasts for Offshore Operations," in WindEurope Conference \& Exhibition, [in press], Bilbao, Spain, 2019. 